\title{
Study protocol and baseline characteristics of newly induced dialysis patients: a prospective multi-center cohort study with biological sample bank, the Ibaraki Dialysis Initiation Cohort Study (iDIC Study)
}

\author{
Takashi Tawara-lida \\ University of Tsukuba

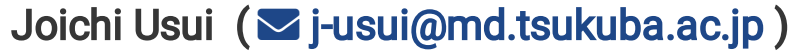 \\ University of Tsukuba \\ Itaru Ebihara \\ Mito Saiseikai General Hospital \\ Takashi Ishizu \\ Tsukuba Central Hospital \\ Masaki Kobayashi \\ Tokyo Medical University Ibaraki Medical Center \\ Yoshitaka Maeda \\ JA Toride Medical Center \\ Hiroaki Kobayashi \\ Ibaraki Prefectural Central Hospital \\ Tokuro Kobayashi \\ Moriya Keiyu Hospital \\ Atsushi Ueda \\ Moriya Keiyu Hospital \\ Makoto Tsuchida \\ Tsuchida Naika Hinyoukika Clinic \\ Shinichiro Sakai \\ Ohta Nephro Clinic \\ Kunihiro Yamagata \\ University of Tsukuba
}

\section{Research Article}

Keywords: dialysis initiation, prospective cohort study, Ibaraki prefecture

Posted Date: May 7th, 2021 
DOI: https://doi.org/10.21203/rs.3.rs-445317/v1

License: (c) (1) This work is licensed under a Creative Commons Attribution 4.0 International License. Read Full License

Version of Record: A version of this preprint was published at BMC Nephrology on March 15th, 2022. See the published version at https://doi.org/10.1186/s12882-022-02729-3. 


\section{Abstract}

Background: Patients with end stage kidney disease (ESKD) face higher risk of life-threatening event including cardiovascular disease. Various risk factors have identified as an agent influencing their life prognosis. The study's purpose was to evaluate the risk factors related to the outcomes of Japanese patients with dialysis induction. In this brief report, we present this cohort study protocol and the baseline characteristics.

Methods: Ibaraki Dialysis Initiation Cohort Study, iDIC Study, is a prospective multi-center cohort study in collaboration with 60 facilities in Ibaraki prefecture, Japan. We collected baseline data from clinical records, blood and urine samples in diabetic nephropathy, hypertensive nephrosclerosis and chronic glomerulonephritis

Results: Sixty tertiary care institutions participated in the iDIC study, and 636 entries were analyzed. In this brief report, we present the baseline data of the entries in a comparison with the data of other similar cohort studies. Of the 636 patients, 424 were males and 212 were females. The average age was $67.4 \pm 13.1$ years old. As the primary kidney disease, 327 cases $(51.4 \%)$ were diagnosed as diabetic nephropathy, 101 cases (15.9\%) as hypertensive nephrosclerosis, and 114 cases (17.9\%) as chronic glomerulonephritis. The average serum creatinine value was $9.1 \pm 2.9 \mathrm{mg} / \mathrm{dL}$, and the average estimated glomerular filtration rate (eGFR) was $5.6 \pm 1.8 \mathrm{~mL} / \mathrm{min} / 1.73 \mathrm{~m} 2$.

Conclusion: We presented the study protocols and baseline characteristics of iDIC Study. Regarding as clinical characteristics of newly induced dialysis patients, the same trend was observed in the other cohort studies. Another study is underway to explore prognostic factors based on the iDIC Study's findings.

\section{Background}

Retarding the progression of chronic kidney disease (CKD) has been a primary endeavor of nephrologists for many years, and dialysis initiation has often been regarded as the main endpoint in this process. Patients with CKD stage G5D, often referred to as End-Stage Kidney Disease (ESKD), have higher risk of cardiovascular disease (CVD) events [1]. Their prognosis is the worst of all CKD stages, sometimes developing into death in the early stage of dialysis initiation. Various efforts have been made to prolong their healthy life expectancy, with investigations into the primary causes of CKD, clinical manifestations and clinical course. These researches have allowed us to identify many risk factors, such as albuminuria, the progression speed of renal dysfunction. These accumulated experiences have been expected to clarify the mechanism of death in dialysis patients and to improve their healthy life expectancy.

Understanding the progression of pre- and post-dialysis CKD has been growing. According to results of Dialysis Outcomes and Practice Patterns Study (DOPPS), Japanese hemodialysis patients had a significantly better long-term survival than those of Europe and the United States, but the short-term (within 6 months) survival did not have a better outcome [2]. Therefore, we need to explore the risk factors 
related with an outcome in Japanese patients with dialysis initiation. In this context, we have planned a prospective multi-center cohort study in a local region, Ibaraki Prefecture, called the Ibaraki Dialysis Initiation Cohort Study, iDIC Study, to analyze baseline data of newly initiated dialysis patients from clinical records, blood and urine samples. In this brief report, we present study protocol and baseline characteristics of iDIC Study.

\section{Methods}

\section{The basic research protocol of the iDIC Study}

The present study was planned as a prospective multi-center cohort study in a specific region, Ibaraki Prefecture, called the Ibaraki Dialysis Initiation Cohort Study, iDIC Study. Sixty tertiary care institutions (universities, referring hospitals and dialysis clinics; see Appendix) participated in this cohort study, enrolling consecutive patients with newly initiated dialysis and following each patient for 24 months. The inclusion criteria of this study were first, consecutive patients with newly initiated dialysis at each participating institute from January 2013 to December 2015; second, patients who needed continuous maintenance dialysis at each participating institute. As the outcome assessment, the primary outcomes of this study were all-cause, cardiovascular and infection-related mortality after dialysis initiation; the secondary outcomes were the rates of admission caused by infection, malignancy and cardiovascular and arteriovenous fistula-related events. In conducting the cohort study, we followed the Declaration of Helsinki and the Ethical Guidelines for Epidemiological Research in Japan. Written informed consent was obtained from each participant for participation, publication, availability of data and materials, and the study protocol was approved by the ethics committee at each participating hospital (the main institute being the University of Tsukuba Hospital, H24-116). This study was registered with the University Hospital Medical Information Network Clinical Trials Registry (UMIN000010806). Patient clinical records and blood and urine samples were collected at baseline. The chief investigators of this cohort (KY), the officer of the research center (JU) and investigators at the top 5 institutes in terms of enrollment numbers (IE, TI, MK, YM, HK) comprised the study's steering committee.

\section{The diagnosis of primary diseases}

The primary cause of ESKD in each participant, e.g., diabetic nephropathy, chronic glomerulonephritis and hypertensive nephrosclerosis, was diagnosed on the basis of medical history and renal biopsy. As the standard clinical practice, the clinical diagnosis of diabetic nephropathy was based on the following criteria: first, the duration of diabetes for 10 years or more, the confirmation of diabetic retinopathy, typically neo-vascular proliferative lesion, and neuropathy, typically sensory disorders of the distal portion of limbs; third, the observation of overt proteinuria, typically nephrotic syndrome; fourth, the exclusion of other primary causes, such as primary glomerulonephritis, other secondary glomerulonephritis including collagen disorder or vasculitis, hereditary kidney disease, cystic kidney disease and drug-induced kidney injury and so on. The clinical diagnosis of hypertensive nephrosclerosis was based on the following criteria: first, the duration of hypertension 10 years or more; second, the lack of overt proteinuria; third, the 
confirmation of hypertensive retinopathy; fourth, the exclusion of other primary causes, such as primary glomerulonephritis, other secondary glomerulonephritis including diabetic nephropathy, collagen disorder or vasculitis, hereditary kidney disease, cystic kidney disease, drug-induced kidney injury and so on.

\section{Baseline characteristics and comorbidities}

Clinical information and laboratory data were collected at study entry via a uniform questionnaire. Baseline information included age, gender, primary cause of ESKD, renal histological diagnosis, complications including diabetes [The International Classification of Diseases, Tenth Revision (ICD-10) codes E10 to E14] and hypertension (ICD-10 codes I10 to I15), prior history of cardiovascular disease and malignancy, kidney disease in family, regular medical checkup, health insurance, motive for consultation, duration between the referral and dialysis initiation, estimated glomerular filtration rate (eGFR) value at 3 time points just before dialysis initiation, duration between operation of arteriovenous fistula to dialysis initiation, and method of continuous dialysis treatment (hemodialysis, peritoneal dialysis or both). Physical status at the baseline included height, body weight, body mass index, blood pressure and daily urine volume. The laboratory data included (from blood) hemoglobin, hematocrit, urea nitrogen, creatinine, albumin, total cholesterol, triglyceride, HDL-cholesterol, LDL-cholesterol, hemoglobin A1c, glucose, uric acid, calcium, phosphate, beta 2-microgloblin, intact-parathyroid hormone, wholeparathyroid hormone and (from urine) protein, blood, urea nitrogen and creatinine. Medication categories at dialysis initiation included angiotensin-converting enzyme inhibitors, angiotensin receptor blockers, beta-blockers, calcium channel blockers, statins, anti-platelet drugs including aspirin, active vitamin D analogues, erythropoiesis-stimulating agents, insulin and other anti-diabetic agents. Prior treatments for primary glomerulonephritis included glucocorticoids, immunosuppressive reagents and tonsillectomy. The qualitative data were reported as number of cases and rate (\%). The quantitative data were written as mean value \pm standard deviation (SD).

\section{Results}

A total of 636 patients were enrolled in the iDIC Study, comprised of 424 males and 212 females, registered from January 2013 to December 2015 (Table). The age of the patients ranged from 17 to 93 years old, with an average age of $67.4 \pm 13.1$ years old. On dialysis initiation, $12.8 \%$ of the patients had a history of renal biopsy, $40.3 \%$ had a medical checkup history, $58.5 \%$ presented with diabetes and $93.8 \%$ with hypertension. Complications prior to dialysis initiation included myocardial infarction (9.6\%), stroke (15.5\%), peripheral artery disease (9.9\%) and malignancy (15.0\%). As the primary kidney disease, 327 cases $(51.4 \%)$ were diagnosed as diabetic nephropathy, 101 cases $(15.9 \%)$ as hypertensive nephrosclerosis and 114 cases $(17.9 \%)$ as chronic glomerulonephritis. Serum creatinine was $9.1 \pm 2.9$ $\mathrm{mg} / \mathrm{dL}$, with eGFR $5.6 \pm 1.8 \mathrm{~mL} / \mathrm{min} / 1.73 \mathrm{~m}^{2}$ at the dialysis initiation.

\section{Discussion}


We have established a prospective multi-center cohort study in a local region, Ibaraki Prefecture, to give clinical evidence of newly initiated dialysis patients. As the center of research, blood and urine samples were collected to investigate biological markers regarding as life-threatening event risk. In this report, we reported the conduct of iDIC Study, and presented baseline clinical data.

The present study showed a similar tendency in the following each finding as the previous three studies, Aichi Cohort Study of Prognosis in Patients Newly Initiated Into Dialysis (AICOPP) [3], The Japanese Society for Dialysis Therapy (JSDT) [4], and the United States Renal Data System (USRDS) [5], reported on the clinical characteristics of newly induced dialysis patients (Table). They had more men than women with an average age of sixties, showing high prevalence of diabetic nephropathy as the primary kidney disease, followed by chronic glomerulonephritis or hypertensive nephrosclerosis. As for prior complications, the percentage of myocardial infarction in the present study was lower than that of other studies, whereas stroke and malignancy accounted for a larger percentage than others. Laboratory findings including hemoglobin, serum uric acid, serum albumin, serum total cholesterol, serum corrected calcium, serum phosphate, other than serum creatinine and eGFR, were almost the same level among the studies. Most strikingly, serum creatinine level and eGFR of the present study were close to the previous two studies from our country, but were lower than that of USRDS, supporting the hypothesis that the timing of dialysis initiation was later in Japan than in the United States. However, the notable point was that even in the United States, mean eGFR at initiation of dialysis is decreasing in recent years, recorded $9.7 \mathrm{ml} / \mathrm{min} / 1.73 \mathrm{~m}^{2}$ in 2016, down from a peak of 10.4 in 2010 [3], reflecting the growing consensus that initiation of dialysis at higher eGFR didn't show a survival benefit.

Here we presented the baseline data of the enrolled patients. The study is now ongoing to assess prognostic factors in these patients, with the ultimate goal of improving life expectancy of dialysis patients.

\section{Conclusions}

We presented the study protocols and baseline characteristics of iDIC Study. Regarding as clinical characteristics of newly induced dialysis patients, the same trend was observed in the other cohort studies. Another study is underway to explore prognostic factors based on the iDIC Study's findings.

\section{Abbreviations}

AICOPP: Aichi Cohort Study of Prognosis in Patients Newly Initiated Into Dialysis; CKD: chronic kidney disease; CVD: cardiovascular disease; DOPPS: Dialysis Outcomes and Practice Patterns Study; ESKD: end stage kidney disease; eGFR: estimated glomerular filtration rate; ICD-10: The International Classification of Diseases, Tenth Revision; iDIC Study: Ibaraki Dialysis Initiation Cohort Study; JSDT: The Japanese Society for Dialysis Therapy; USRDS: the United States Renal Data System.

\section{Declarations}




\section{Ethics approval and consent to participate}

In conducting the cohort study, we followed the Declaration of Helsinki and the Ethical Guidelines for Epidemiological Research in Japan. Written informed consent was obtained from each participant for participation, availability of data and materials, and the study protocol was approved by the ethics committee at each participating hospital (the main institute being the University of Tsukuba Hospital, H24-116). This study was registered with the University Hospital Medical Information Network Clinical Trials Registry (UMIN000010806).

\section{Consent for publication}

Written informed consent was obtained from each participant for publication.

\section{Availability of data and materials}

The datasets used and/or analyzed during the current study are available from the corresponding author on reasonable request.

\section{Competing interests}

None.

\section{Funding}

This work was supported by grants from the Research Committee of Intractable Renal Disease of the Ministry of Health, Labour, and Welfare of Japan and a Grant-in-Aid for Research on Advanced Chronic Kidney Disease (REACH-J), Practical Research Project for Renal Diseases from Japan Agency for Medical Research and development, AMED.

\section{Author's contributions}

The chief investigators of this cohort (KY), the officer of the research center (JU) and investigators (IE, TI, MK, YM, HK) comprised the study's steering committee. Steering committee members contributed to patient entries, design of the study, data analysis and writing manuscript. Others (TK, AU, MT, SS) contributed to patient entries. All authors read and approved the submission.

\section{Acknowledgements}

We thank the members of the Research Center of the iDIC Study, Dr. Takamoto Yanagawa, Ms Yuko Sudo, Ms Rie Kikkou, Ms Mikie Daigo and Ms Hideko Sakurai, for their technical support.

\section{Authors' information}

${ }^{1}$ Department of Nephrology, Faculty of Medicine, University of Tsukuba, Tsukuba, Ibaraki, Japan.

${ }^{2}$ Department of Nephrology, Mito Saiseikai General Hospital, Mito, lbaraki, Japan. ${ }^{3}$ Department of Renal 
and Dialysis Medicine, Tsukuba Central Hospital, Ushiku, Ibaraki, Japan. ${ }^{4}$ Department of Nephrology, Tokyo Medical University Ibaraki Medical Center, Ami, Japan. ${ }^{5}$ Nephrology Division, Department of Internal Medicine, JA Toride Medical Center, Toride, Japan. ${ }^{6}$ Department of Nephrology, Ibaraki Prefectural Central Hospital, Kasama, Japan. ${ }^{7}$ Moriya Keiyu Hospital, Moriya, Japan. ${ }^{8}$ Department of Nephrology, Hitachi General Hospital, Hitachi, Japan. ${ }^{9}$ Tsuchida Naika Hinyoukika Clinic, Hitachinaka, Japan. ${ }^{10}$ Ohta Nephro Clinic, Hitachiohta, Japan. *present address: Department of Nephrology, Ushiku Aiwa General Hospital, Ushiku, Ibaraki, Japan.

\section{References}

1. Gansevoort RT, Correa-Rotter R, Hemmelgarn BR, Jafar TH, Heerspink HJ, Mann JF, et al. Chronic kidney disease and cardiovascular risk: epidemiology, mechanisms, and prevention. Lancet 2013; 382: 339-352.

2. Goodkin DA, Bragg-Gresham JL, Koenig KG, Wolfe RA, Akiba T, Andreucci VE, et al. Association of comorbid conditions and mortality in hemodialysis patients in Europe, Japan, and the United States: The Dialysis Outcomes and Practice Patterns Study (DOPPS). J Am Soc Nephrol 2003; 14(12): 32703277.

3. Hishida M, Tamai H, Morinaga T, Maekawa M, Aoki T, Tomida H, et al. Aichi cohort study of the prognosis in patients newly initiated into dialysis (AICOPP): baseline characteristics and trends observed in diabetic nephropathy. Clin Exp Nephrol. 2016; 20(5): 795-807.

4. Nitta K, Masakane S, Hanafusa N, Taniguchi M, Hasegawa T, Nakai S, et al. Current situation for dialysis therapy in Japan - 1 January-31 December 2017. J. Jpn. Soc. Dial. Ther. 2018; 51: 699766.

5. United States Renal Data System. 2018 USRDS annual data report: Epidemiology of kidney disease in the United States. National Institutes of Health, National Institute of Diabetes and Digestive and Kidney Diseases, Bethesda, MD, 2018. https://www.usrds.org/adr.aspx. Accessed 6 Aug 2019.

\section{Table}

Table. Comparion of clinical characteristics at entry in dyalisis indcution cohort studies 
year

\begin{tabular}{ccc} 
iDIC study & AICOPP & JSDT \\
\hline $2013-2015$ & $2011-2013$ & 2006 \\
636 & 1,524 & 9,689 \\
$614 / 22 / 0$ & $1,409 / 105 / 0$ & $8,702 / 443 / 0$ \\
$67.4 \pm 13.1$ & $67.5 \pm 13$ & $66.6 \pm 13.2$ \\
$424 / 212$ & $1,032 / 492$ & $6241 / 3448$ \\
12.8 & $\mathrm{NA}$ & $\mathrm{N} / \mathrm{A}$ \\
40.3 & $\mathrm{NA}$ & $\mathrm{NA}$ \\
58.5 & 53.3 & 58.8 \\
93.8 & $\mathrm{NA}$ & $\mathrm{NA}$ \\
9.6 & 15.8 & 8.0 \\
15.5 & 13.7 & 15.0 \\
9.9 & 4.7 & $\mathrm{NA}$ \\
15.0 & 10.6 & 7.3 \\
10.3 & $\mathrm{NA}$ & $\mathrm{NA}$
\end{tabular}

Primary kidney disease (\%)

Diabetic nephropathy

Hypertensive nephrosclerosis

Chronic glomerulonephritis

Autosomal dominant polycystic kidney disease

Rapidly progressive glomerulonephritis

Other disease

Unknown cause

$\begin{array}{ccc}51.4 & 43.2 & 42.9 \\ 15.9 & 25.3 & 9.4 \\ 17.9 & 14.7 & 25.6 \\ 2.5 & 3.3 & 2.4 \\ 2.5 & \text { NA } & 1.2 \\ 6.0 & 13.5 & 8.6 \\ 3.8 & & 9.9\end{array}$

Body mass index $\left(\mathrm{kg} / \mathrm{m}^{2}\right)$

$24.3 \pm 4.5$

$23.5 \pm 4.4$

NA

Systolic blood pressure ( $\mathrm{mmHg}$ )

$150.6 \pm 26.5$

$151.1 \pm 26.0$

$153 \pm 28$

Diastolic blood pressure $(\mathrm{mmHg})$

Hemoglobin $(\mathrm{g} / \mathrm{dL})$

$77.8 \pm 16.3$

$77.1 \pm 15.0$

$78 \pm 16$

Serum creatinine $(\mathrm{mg} / \mathrm{dL})$

$9.0 \pm 1.8$

$9.4 \pm 1.5$

$8.4 \pm 1.6$

Estimated glomerular filtration rate

$9.1 \pm 2.9$

$9.0 \pm 3.2$

$8.2 \pm 3.3$

$\left(\mathrm{mL} / \mathrm{min} / 1.73 \mathrm{~m}^{2}\right)$

Serum uric acid $(\mathrm{mg} / \mathrm{dL})$

$5.6 \pm 1.8$

$5.6 \pm 2.2$

$5.5 \pm 3.3$

Serum albumin $(\mathrm{g} / \mathrm{dL})$

$8.5 \pm 8.6$

$8.8 \pm 2.4$

NA

Serum total cholesterol $(\mathrm{mg} / \mathrm{dL})$

$3.5 \pm 2.6$

$3.2 \pm 0.6$

$158.5 \pm 48.0 \quad 162 \pm 44.3$

$3.3 \pm 0.6$

Serum corrected calcium (mg/dL)

Serum phosphate $(\mathrm{mg} / \mathrm{dL})$

Hemoglobin A1c (\%)

$8.5 \pm 1.0$

$8.6 \pm 1.1$

NA

Intact-parathyroid hormone $(\mathrm{pg} / \mathrm{ml})$

$6.2 \pm 1.9$

$6.4 \pm 1.9$

$8.6 \pm 1.1$

$5.9 \pm 0.9$

$5.8 \pm 0.9$

$5.8 \pm 1.9$

$289.5 \pm 224.5 \quad 353 \pm 292.4$

NA

NA

Abbreviations: iDIC Study; the Ibaraki Dialysis Initiation Cohort Study,

AICOPP; Aichi Cohort Study of Prognosis in Patients Newly Initiated Into Dialysis, JSDT; The Japanese Society for Dialysis Therapy, USRDS; the United States Renal Data System,

HD; Hemodialysis, PD; Peritoneal dialysis, NA; not available. 


\section{Supplementary Files}

This is a list of supplementary files associated with this preprint. Click to download.

- iDICstudyAppendixforBMCsubmit.docx 